\title{
Supporting Information \\ Rapid volatilization induced mechanically robust shape-morphing structures toward 4D printing
}

Qiang Zhang ${ }^{\$ 1,2}$, Xiao Kuang ${ }^{\$ 1}$, Shayuan Weng ${ }^{1,3}$, Zeang Zhao ${ }^{2}$, Haosen Chen $^{4}$, Daining Fang*2,4 H. Jerry $\mathrm{Qi}^{* 1}$

${ }^{1}$ The George W. Woodruff School of Mechanical Engineering

Georgia Institute of Technology, Atlanta, GA 30332, USA

${ }^{2}$ State Key Laboratory for Turbulence and Complex System, College of Engineering, Peking University, Beijing, 100871, People's Republic of China

${ }^{3}$ College of Aerospace Engineering, Chongqing University, Chongqing, 400044, People's Republic of China

${ }^{4}$ Institute of Advanced Structure Technology, Beijing Institute of Technology, Beijing, 100081, People's Republic of China

${ }^{\$}$ These authors contributed equally to this work.

*Corresponding author. Email: fangdn@bit.edu.cn (D.F.); qih@me.gatech.edu (H.J.Q.) 


\section{Photopolymerization reaction}

A

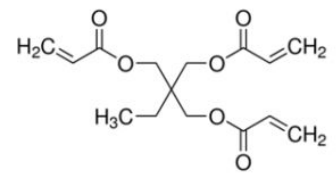

Trimethylolpropane triacrylate (TMPTA)
B

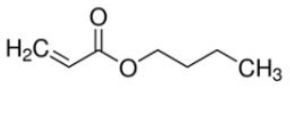

Butyl acrylate (BA)
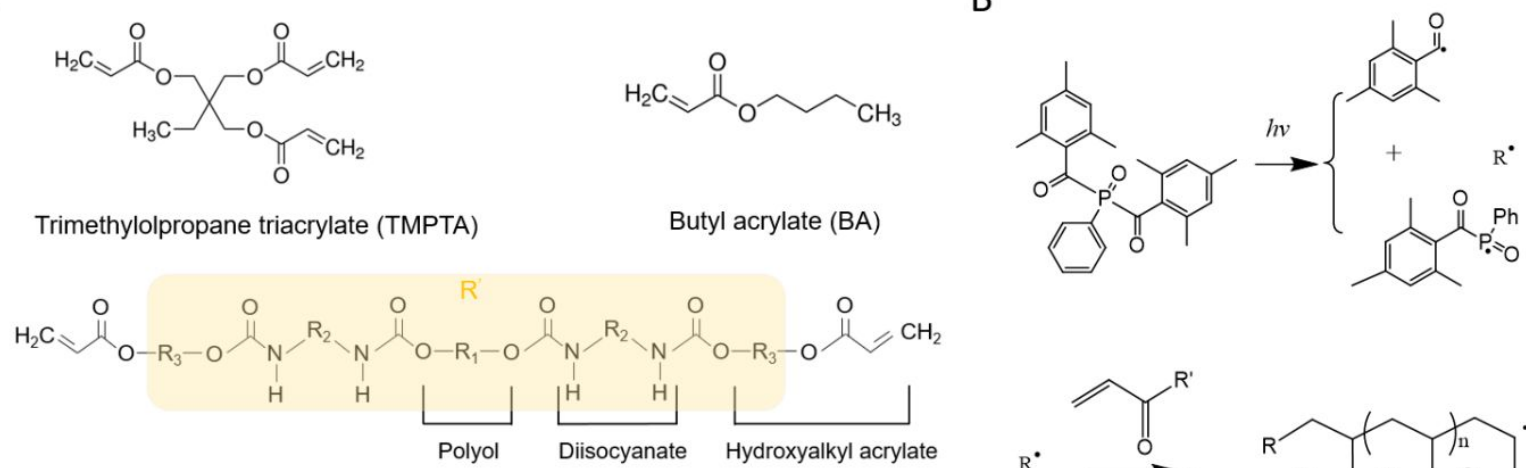

Aliphatic urethane diacrylate (AUD)

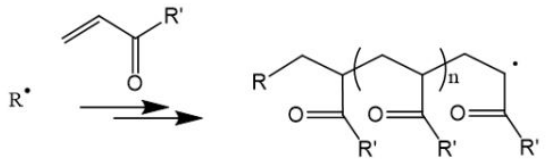

C
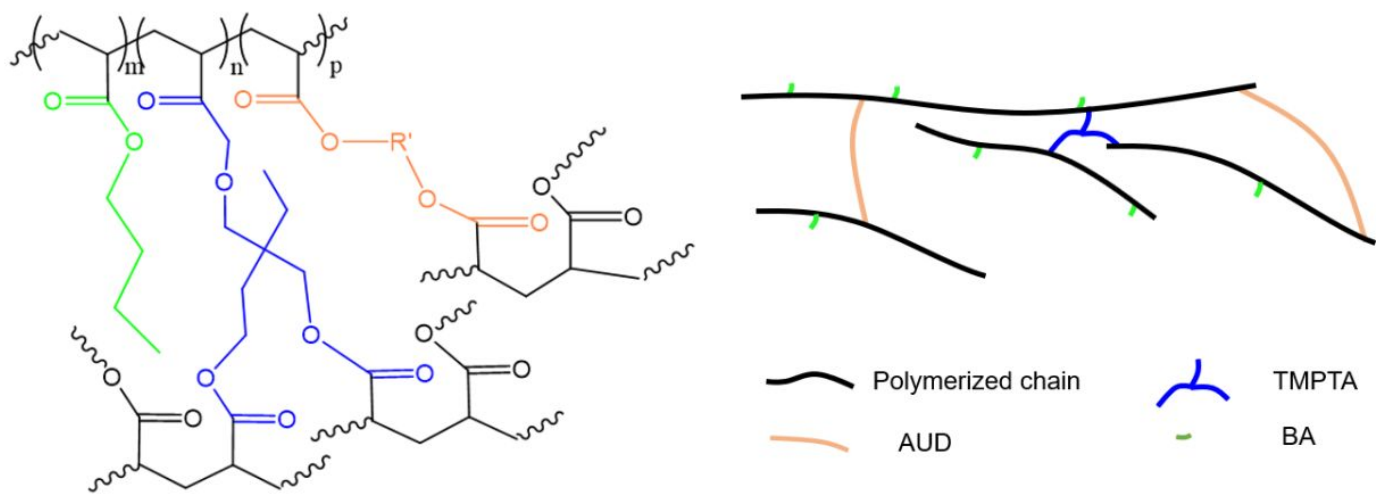

Figure S1. Chemical structure of polymers and reaction process. (A) Chemical structure of molecules participating in the reaction. (B) Reaction process of the free-radical reaction. (C) Schematic of the chemical structure of the cured polymer network.

The material system used in this work contains trimethylolpropane triacrylate (TMPTA), aliphatic urethane diacrylate (AUD; Ebecryl 8402), and n-butyl acrylate (BA). Figure S1A shows the main structures of the three acrylic monomers and oligomers, namely, TMPTA, BA, and AUD. ${ }^{1}$ Among them, BA is a monofunctional acrylate acting as the chain extender; while AUD (diacrylate) and TMPTA (triacrylate) containing multiple carbon-carbon double bonds $(\mathrm{C}=\mathrm{C})$ are cross-linkers. The photo-polymerization is a chain-growth polymerization reaction in which the free radicals generated by photoinitiators initiate the rapid radical polymerization between the carbon-carbon double bond $(\mathrm{C}=\mathrm{C})$. The ester bonds $(\mathrm{C}=\mathrm{O})$ in both the monomer and oligomer do not change during the photopolymerization (Figure $\mathrm{S} 1 \mathrm{~B}$ ). $\mathrm{C}=\mathrm{C}$ is the only source of curing and $\mathrm{C}=\mathrm{O}$ is preserved during the curing reaction. Therefore, the $\mathrm{C}=\mathrm{O}$ stretching vibration peak at frequency $1725 \mathrm{~cm}^{-1}$ was used as the standard to calculate the reaction conversion of the double 
bond. The left image in Figure S1C shows the chemical structure of the cured polymer network while the right image shows the schematic of the chemical structure of the cured polymer network.

\section{Material volatile properties}

Strictly speaking, any substance (including liquid and solid) has a tendency to volatilize into a gaseous state. However, a substance with a high vapor pressure at normal temperatures is often referred to as volatile. According to the suppliers, the vapor pressure of TMPTA is $<0.01 \mathrm{mmHg} @ 20.00{ }^{\circ} \mathrm{C}$, while Ebecryl 8402 is described as "Low/zero VOC (Volatile Organic Compounds)". ${ }^{3}$ By contrast, BA used in this work is $4 \mathrm{mmHg} @ 20^{\circ} \mathrm{C}$. Therefore, both TMPTA and Ebecryl 8402 have a low vapor pressure and their evaporation can be ignored compared with that of BA. To an adequate approximation, BA is the only volatile species here.

\section{Photopolymerization kinetics}

During the experiments, the photocurable resin is cured under the irradiation of a UV light irradiated from one side (top or bottom) which is perpendicular to the resin surface. Upon illuminating, the curing front propagates along the depth direction starting from the surface that is closer to the UV light. Here, we adopt the model used in previous researches to describe the reaction kinetics of the resin used in our experiments. ${ }^{4-}$

${ }^{6}$ In the model, the exposure time $t$ and UV light intensity $I_{0}$ can be used interchangeably, as the front kinetics is controlled by their product (light dose, $d=t \times I_{0}$ ). According to the model, the normalized reaction conversion can be described as

$$
\varphi(z, t)=1-\exp \left[-K I_{0} \exp (-\mu z) t\right]
$$

where $\varphi(z, t)$ is the normalized reaction conversion $(0<\varphi<1), \mathrm{K}$ is the overall reaction conversion rate, $\mu$ is the light attenuation coefficient, and $z$ is the distance from the illuminating surface, respectively. The front position $z_{f}$, corresponding to the solidified thickness, is given by

$$
Z_{f}=\frac{\ln \left[\frac{{ }^{K I_{0} t}}{\ln \left(\frac{1}{1-\varphi_{c}}\right)}\right]}{\mu}
$$

where $\varphi_{c}$ is the critical conversion fraction corresponding to the gel point of the used material system. In Eq. S1, $K$, and $\mu$ are dependent on the specified photocurable material systems. In our experiments, they can be determined through fitting the measured curing front position and the exposure time according to Eq. S2.

\section{Mechanics of the bending behavior of a self-morphing rectangular strip}


After a rectangular strip is cured under the exposure of a UV light, a gradient of reaction conversion as well as residual BA along its thickness direction will exist in the resulted network. Due to this gradient, the strip will bend upon volatilization of BA. The dynamic bending curvature during the volatizing process depends on a combination of printing parameters (curing time $t$, grayscale value $G$ ), geometry (thickness $h$ ), and volatizing conditions (temperature $T$ ). Note that volatilization is a complex physical process and the rate of volatilization can be influenced by many factors (such as temperature, humidity, air circulation), we here only consider temperature and ignore other influencing factors. Based on this simplification, the bending behavior of a strip is time dependent as well as temperature dependent. We build a theoretical model in the framework of beam theory to capture these features. To further simplify the analysis, heat transfer and diffusion coupled deformation are not considered.

After photopolymerization, the molar concentration of the residual BA in the network is denoted as $C$ $(z, t)$. At time $t=0$, the residual BA starts to volatilize out of the network and $C(z, t)$ will decrease. As the thickness $(h)$ of the strip is relatively thin, we can assume that at time $t, C(z, t)$ can be linked to the initial concentration at position $z$ by an exponential function. Therefore $C(z, t)$ can be given by

$$
C(z, t)=C(z, 0) \exp (-D t)
$$

where $C(z, 0)$ is the concentration of the unreacted BA at the beginning of the volatilizing process, which can be determined by the normalized reaction conversion $\varphi$, and the initial total molar concentration of BA $C_{B A}$ before reaction

$$
C(z, 0)=(1-\varphi(z)) C_{B A}
$$

In Eq. S3, $D$ is a temperature-dependent factor which is used to characterize the volatilizing rate of BA. During experiments, we note that the volatilizing rate is highly sensitive to temperature. A higher temperature is always companied by a higher volatilizing rate. As volatilization is one of thermal-induced processes, we can simply use an Arrhenius' law to describe the temperature dependent volatilizing rate as ${ }^{7}$

$$
D=D_{0} \exp \left(\frac{-E_{a}}{R T}\right)
$$

where $D_{0}$ is the pre-exponential factor, a constant for each volatilizing process, $E_{a}$ is the activation energy for the process, and $R$ is the universal gas constant. Here, both $D_{0}$ and $E_{a}$ can be determined through experiments.

The volatilization-induced shrinking stretch $\lambda_{s}$ during the deformation is given by

$$
\lambda_{s}=(1+\Omega \Delta C)^{\frac{1}{3}}
$$

where $\Omega$ is the molar volume of BA, and $\Delta C$ is the molar concentration of the loss of BA at time t, determined by $\Delta C(z, t)=C(z, t)-C(z, 0)$. For a long enough time, the residual $\mathrm{BA}$ in the network will 
approach zero, which represents a final equilibrium state of the dynamic volatizing process. By solving Eq. S3, Eq. S4, and Eq. S6 at the equilibrium state, we obtain

$$
\lambda_{s}=\left(1-\Omega \mathrm{C}_{B A}(1-\varphi)\right)^{\frac{1}{3}}
$$

At the equilibrium state, if we ignore the density variation before and after photopolymerization, the volatilization induced volume shrinkage ratio should satisfy

$$
1-\alpha=m(1-\varphi)
$$

where $\alpha$ and $m$ are the volume shrinkage ratio and weight ratio (wt \%) of BA, respectively. Considering that $\alpha=\lambda_{s}{ }^{3}$, the product $\Omega C_{B A}$ of the coefficients $\Omega, C_{B A}$ in Eq. S6 can be determined to be equal to $m$. Now, combining Eq. S3-8, Eq. S6 becomes

$$
\lambda_{s}=\left(1-m(1-\varphi)\left(1-\exp \left(-D_{0} \exp \left(\frac{-E_{a}}{R T}\right) t\right)\right)\right)^{\frac{1}{3}}
$$

According to beam theory, the elastic strain, $\varepsilon$, in the strip is given as

$$
\varepsilon=\kappa\left(z_{n}-z\right)-\ln \lambda_{s}
$$

Here, $\kappa$ is the curvature of the neutral surface of the strip, and $z_{n}$ is the position of the neutral surface. During the self-morphing process of a strip, the equilibrium force and moment should satisfy

$$
\begin{gathered}
\sum F=\int_{0}^{h} \sigma d z=0 \\
\sum M=\int_{0}^{h} \sigma \varepsilon d z=0
\end{gathered}
$$

To fully solve the equations above, we still need to know the profile of Young's modulus along the thickness direction. Note that Young's modulus is dependent on reaction conversion and the profile of conversion along the thickness direction can be determined according to Eq. S1, we fitted the experimental results of Young's modulus versus normalized conversion using an empirical relationship based on previous investigations $\mathbf{s}^{8,9}$

$$
E(\varphi)=E_{0} \exp \left(a\left(\varphi-\varphi_{c}\right)\right)
$$

where $E_{0}, a$ are the fitting parameters and can be determined through experiments.

With all the determined parameters, the deformation of a bending strip can be determined through solving Eq. S1 and Eq. S9-13.

\section{Temperature influence on the bending behavior of a self-morphing rectangular strip}



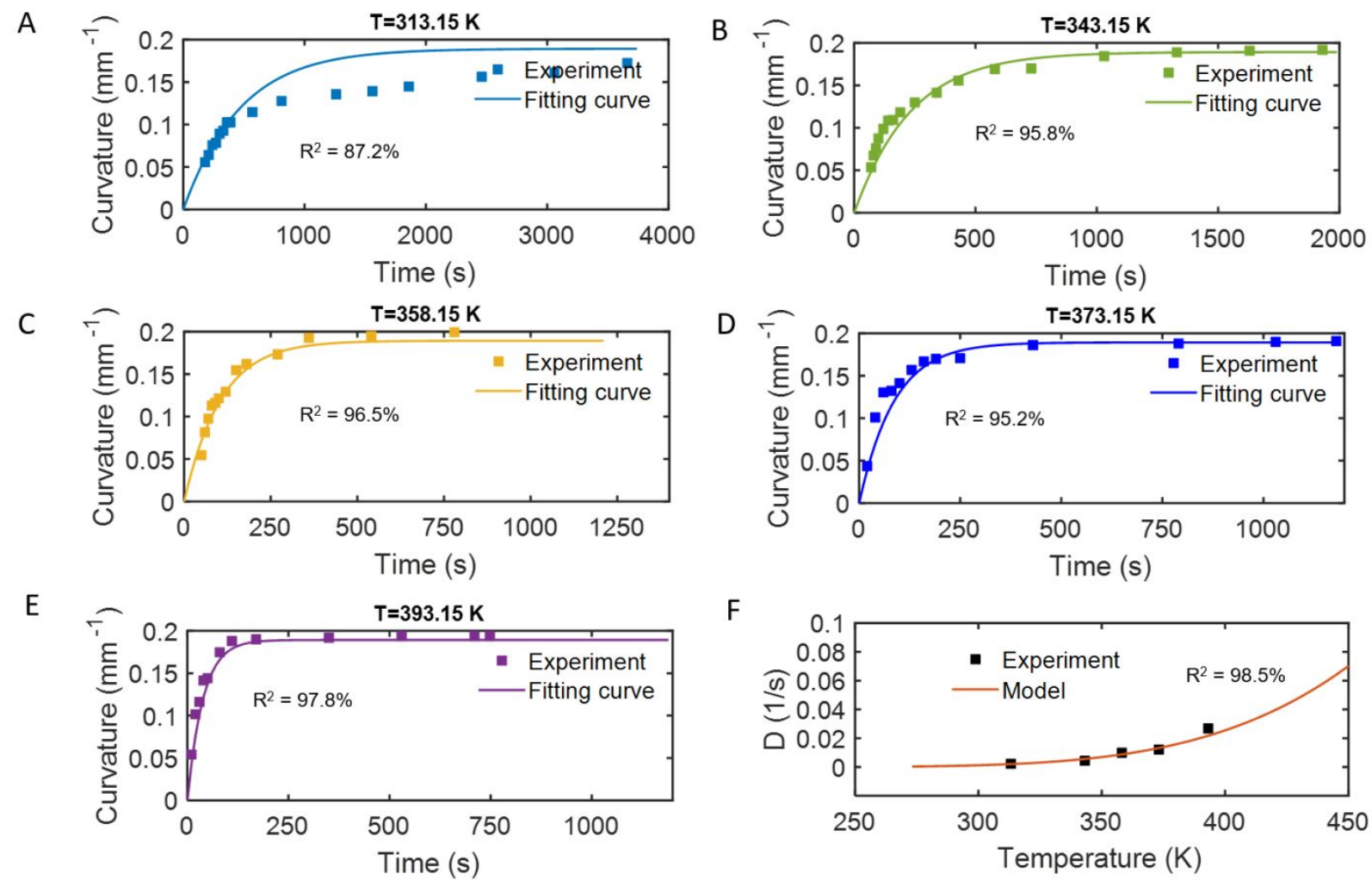

Figure S2. Temperature-dependent bending process of a rectangular strip. Bending curvature as a function of time measured at (A) $40{ }^{\circ} \mathrm{C},(\mathbf{B}) 70^{\circ} \mathrm{C},(\mathbf{C}) 85^{\circ} \mathrm{C}$, (D) $100{ }^{\circ} \mathrm{C}$, and (E) $120^{\circ} \mathrm{C}$, respectively. Experimental results are fitted by the model to find the best temperature-dependent factor $\mathrm{D}$ for each temperature. (F) The fitted temperature-dependent factor as a function of temperature. Dots represent experimental results while the curve represents analytic results. The R-squared assessment for the fitting curves is shown in each figure.

As the volatilization process can be accelerated by higher temperature, it is necessary to investigate the temperature influence on the bending behavior of a self-morphing rectangular strip. We fabricated a group of samples $(10 \times 2.5 \times 0.19 \mathrm{~mm})$ by using a UV light of $7.16 \mathrm{~mW} / \mathrm{cm}^{2}$ and an exposure time of $20 \mathrm{~s}$ and let the samples bend at different temperatures $\left(40^{\circ} \mathrm{C}, 70{ }^{\circ} \mathrm{C}, 85^{\circ} \mathrm{C}, 100{ }^{\circ} \mathrm{C}\right.$, and $\left.120^{\circ} \mathrm{C}\right)$. During the bending process, we recorded the bending curvatures versus time as shown in Figure S2A-E. As the temperature increases, the bending speed increases, and less time is required to complete the bending process. Compared with the strip bending at $40^{\circ} \mathrm{C}$ which needs $\sim 2000 \mathrm{~s}$ to achieve its final curvature, the strip bending at 120 ${ }^{\circ} \mathrm{C}$ only requires $\sim 200 \mathrm{~s}$ to achieve its final curvature, demonstrating a 10-fold acceleration. In addition, we observed that temperature has no obvious influence on the final curvature that the strips can achieve. Figure S2A-E also show the fitted curves with a fitting parameter of $D$ (Eq. S5) according to the model above. With different values of $D$ at different temperatures, the parameter $D_{0}$ and $E_{a}$ in Eq. S5 can be determined to be $233.50 \mathrm{~s}^{-1}$ and 3.0e4 J/mol, respectively. Results are shown in Figure S2F. In our experiments, the temperature has a slight effect on Young's modulus of the material. For a rubbery material, the modulus 
will increase with temperature (following a relationship $E=3 n k T$ where $n$ is chain density and $k$ is Boltzmann's constant, respectively). For our material system, the modulus $E$ at a temperature $T$ is obtained by multiplying the temperature ratio $\left(\frac{T}{T_{\text {base }}}\right)$ with the base modulus $\left(E_{\text {base }}\right)$ measured at room temperature ( $\left.T_{\text {base }}\right)$ via relation $E=\frac{T}{T_{\text {base }}} E_{\text {base }}$.

\section{Correlation between grayscale value and light intensity}

During our experiments, we adjust the UV light intensity of a projected pattern by controlling its grayscale value. Here, the grayscale value is selected from the standard RGB value range $(0-255)$. In order to establish the relationship between light intensity and grayscale value, we experimentally measured the UV light intensity as a function of grayscale value from 23 to 255. The results are shown in Figure 1B. We found that the relationship can be well fitted by a Gaussian function

$$
I_{0}=I_{M} e^{-\frac{(g-a)^{2}}{2 b^{2}}}
$$

where $I_{M}$ is the light intensity at a grayscale of 255, $a$, and $b$ are fitting parameters, respectively. In our experiments, $I_{M}=19.38 \mathrm{~mW} / \mathrm{cm}^{2}, a=269.65, b=86.34$, and the fitted curve is also plotted in Figure 1B. From Figure 1B, the light intensity of a 149 grayscale UV light can be determined to be $7.16 \mathrm{~mW} / \mathrm{cm}^{2}$. 
A
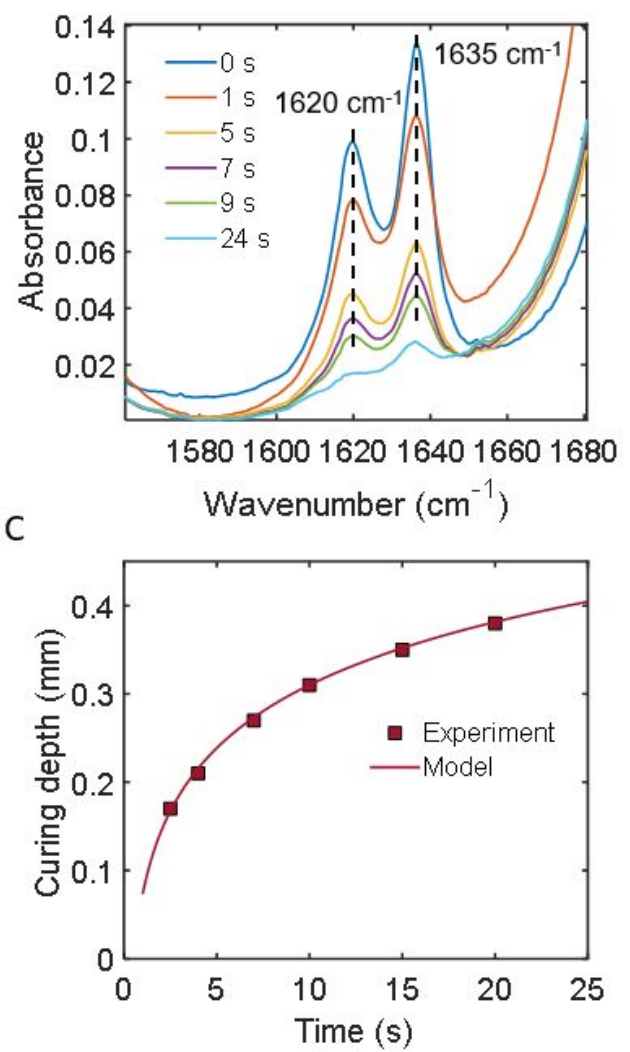

$\mathrm{E}$

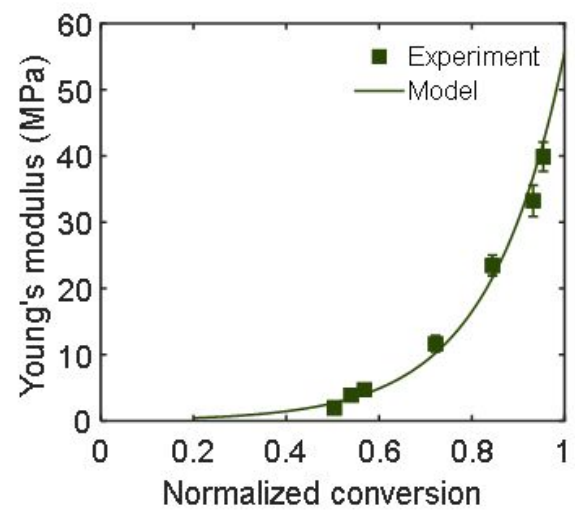

B

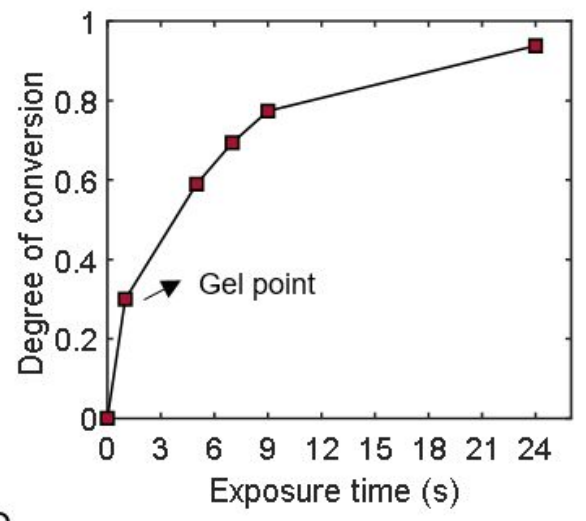

D

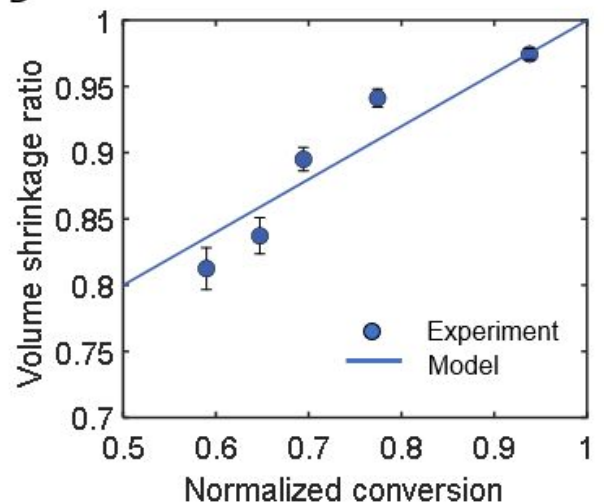

$\mathrm{F}$

$6 \mathrm{~s} \quad 10 \mathrm{~s} \quad 20 \mathrm{~s}$

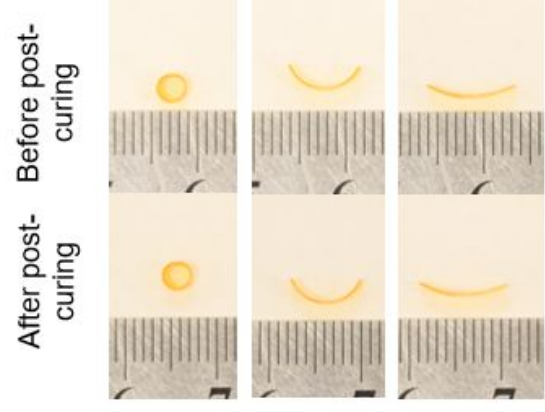

Figure S3. Properties of the photocurable resin during photopolymerization. (A) FTIR spectrums of samples cured for different times. (B) Degree of conversion as a function of exposure time. (C) Curing depth as a function of time. (D) Volume shrinkage ratio as a function of normalized conversion. (E) Young's modulus as a function of normalized conversion. (F) The shape of the rectangular strips with different curing time $(6 \mathrm{~s}, 10 \mathrm{~s}$, and $20 \mathrm{~s})$ before and after post-curing.

\section{Properties of the photocurable resin during photopolymerization}

In order to experimentally determine the reaction conversion of a polymer cured under different conditions, we fabricated samples (a small strip with a thickness of $0.10 \mathrm{~mm}$ ) with different reaction conversion by exposing resins without photo absorbers with a $7.16 \mathrm{~mW} / \mathrm{cm}^{2} \mathrm{UV}$ light for different times $(0 \mathrm{~s}, 1 \mathrm{~s}, 5 \mathrm{~s}, 7 \mathrm{~s}$, 
$9 \mathrm{~s}, 24 \mathrm{~s}$ ). These samples were then transferred to a Fourier transform infrared (FTIR) spectrometer (Nicolet iS50, Thermo Scientific, Waltham, MA, USA) to obtain the conversion $(\Phi)$ of $\mathrm{C}=\mathrm{C}$ double bonds by averaging 32 scans of the signal at a resolution of $2 \mathrm{~cm}^{-1}$ in attenuated total reflectance mode. FTIR spectrums are shown in Figure S3A. By calculating the decrease of the area under the doublet of each curve,

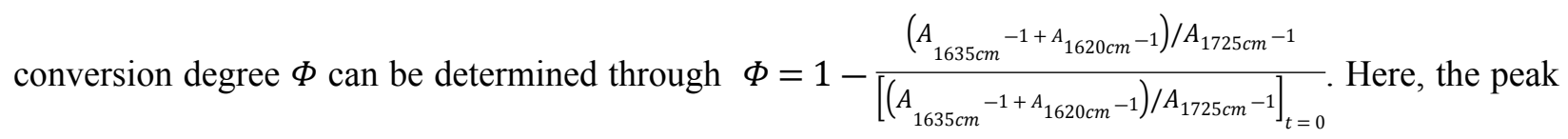
at $1725 \mathrm{~cm}^{-1}$ for $\mathrm{C}=\mathrm{O}$ bonds was used as a reference as it is stable during the reaction. For a better theoretical analysis, the conversion obtained here was normalized to be $\varphi=\Phi / \Phi_{\max }$. In Figure S3B, we report the degree of conversion as a function of exposure time derived from the FTIR data shown in Figure S3A. During experiments, we found that for an exposure time lower than $1 \mathrm{~s}$, the resin cannot be cured into a solid because of low conversion; while for an exposure time equaling to $1 \mathrm{~s}$, the resin is cured into a solid but in a slimy and not fully formed state. For longer exposure time, the solid becomes denser and more well cured. Therefore, the exposure time for $1 \mathrm{~s}$ roughly corresponds to the gel point of the resin. We checked the degree of conversion at this point in Figure S3B and found that it equals to $\sim 0.3$. We thus determine $\sim 0.3$ as the critical conversion fraction for this polymer system.

In order to characterize the curing kinetics of the resin, we used the UV light with a light intensity of $7.16 \mathrm{~mW} / \mathrm{cm}^{2}$ to cure resins from the bottom with different exposure time (from $4 \mathrm{~s}$ to $22 \mathrm{~s}$ ). During each exposure, the curing front propagates from the bottom until achieving a depth which is dependent on the curing time. After each curing, we measured the thickness (curing depth) of the cured samples. By fitting the curing depth versus time, parameters $K$ and $\mu$ in our experiments are determined to be $0.09 \mathrm{~cm}^{2} /(\mathrm{mWs})$ and $9.71 \mathrm{~mm}^{-1}$, respectively. Both the experimental results and the fitted curve are shown in Figure S3C.

To determine how much BA can volatilize for a specified reaction conversion after curing, samples (10 $\mathrm{mm} \times 2.5 \mathrm{~mm} \times 0.1 \mathrm{~mm}$ ) were fabricated by curing resins without photo absorbers for a different time under the same light intensity $\left(7.16 \mathrm{~mW} / \mathrm{cm}^{2}\right)$. After curing, the weights of these cured samples were measured by a balance. The samples were then transferred into an oven (Fisher Scientific) to remove the residual BA in the network. After the total loss of BA ( 3 hours at $60^{\circ} \mathrm{C}$ temperature), the weights of the samples were measured again. The volume shrinkage ratio $V / V_{0}$ can be obtained from the weight loss. Both experimental and analytical (according to Eq. S8) results can be seen in Figure S3D.

To obtain Young's modulus of the materials with different reaction conversion before and after postcuring, we fabricated two sets of samples $(\sim 25 \mathrm{~mm} \times 4.5 \mathrm{~mm} \times 0.1 \mathrm{~mm})$ by curing resins without photo absorbers for a different time under the same light intensity $(7.16 \mathrm{~mW} / \mathrm{cm} 2)$. After curing all samples were transferred into the oven to remove the residual BA in the network. After the total loss of BA (3 hours at $60{ }^{\circ} \mathrm{C}$ temperature), one set of the samples was post-cured under a light intensity of $30 \mathrm{~mW} / \mathrm{cm} 2$ for 15 minutes while the other received no modification. Then we used a DMA tester (model Q800, TA 
Instruments) to obtain the stress-strain curves of all samples in a strain mode. Results of the batch without post-curing are shown both in Figure S3E and Figure 5A while the results of the batch with post-curing are shown only in Figure 5A. The fitting parameters $E_{0}$ and $a$ are determined to be $1.066 \mathrm{MPa}$ and 6.086 , respectively.

For the material system used here, volume shrinkage originates from the distance decrease when the carbon-carbon double bonds $(\mathrm{C}=\mathrm{C})$ convert to carbon-carbon single bonds $(\mathrm{C}-\mathrm{C})$ during curing. Strictly speaking, the volume shrinkage associated with the photopolymerization during the post-curing process affects the formed 3D structure. However, in our work, the volume shrinkage during the second photocuring is very small. This is because before post-curing the formed 3D structures already have an initial reaction conversion fraction which is typically in the range of $0.55 \sim 0.9$ (Figure S3D). This means that more than half of the $\mathrm{C}=\mathrm{C}$ in the cured structures have been already converted to $\mathrm{C}-\mathrm{C}$ in the printing process. After the volatilization of residual BA (which introduced some volume shrinkage), the second photocuring consumes the residual $\mathrm{C}=\mathrm{C}$ bonds but has negligible volume shrinkage. We validate this by printing three rectangular strips $(10 \times 2.5 \times 0.19 \mathrm{~mm})$ with different exposure time, namely, $6 \mathrm{~s}, 10 \mathrm{~s}$, and $20 \mathrm{~s}$. The intensity of the UV light was fixed at $7.16 \mathrm{~mW} / \mathrm{cm}^{2}$ and the light was shined from one side of the strip. After printing, there was a conversion gradient along the thickness direction of the strip and the initially straight strips bent into a curved shape after the volatilization of BA (the top panel of Figure S3F). The curvature of the three bent strips was measured to be $0.68 \mathrm{~mm}^{-1}, 0.25 \mathrm{~mm}^{-1}$, and $0.08 \mathrm{~mm}^{-1}$, respectively. After post-curing, the curvature of the rectangular strips was measured again (the bottom panel of Figure $\mathrm{S} 3 \mathrm{~F}$ ) and they are $0.67 \mathrm{~mm}^{-1}, 0.24 \mathrm{~mm}^{-1}$, and $0.08 \mathrm{~mm}^{-1}$, respectively. We found that for all the three strips with initial conversion fraction from low $(\sim 0.6)$ to high $(\sim 0.9)$, the curvature measured before and after post-curing is almost the same. Therefore, the volume shrinkage associated with the second polymerization of the residual non-volatile unreacted monomers during the post-curing process contributes little to the shape change.

\section{Geometry analysis of folding a 2D hexagonal sheet into a buckyball structure}




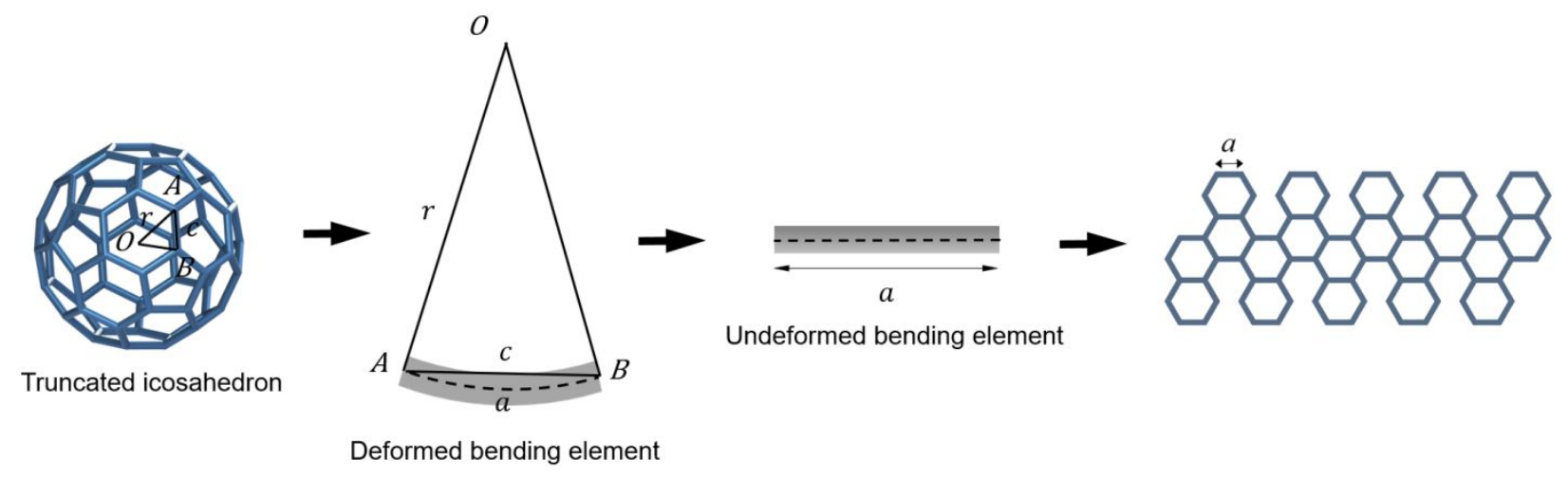

Figure S4. Schematic illustration of the geometry analysis of folding a 2D hexagonal sheet into a Buckyball structure.

For a 2D hexagonal sheet that will fold into a buckyball structure (Figure S4), each edge of the hexagons bends with the same curvature $\mathrm{K}$, which satisfies

$$
\kappa=\frac{1}{r}
$$

where $r$ is the distance between a vertex and the center of the folded truncated icosahedron (length of OA). Note that $r$ is also the radius of the circumscribed sphere of that truncated icosahedron. Based on a simple geometry analysis, it is not difficult to find that the edge length of a truncated icosahedron can be determined by its circumscribed sphere radius

$$
r \approx 2.478 c
$$

where $c$ is the distance between a pair of two neighboring vertices in a truncated icosahedron (length of $\mathrm{AB})$. The arc length of $\mathrm{AB}$, which is also the length of the undeformed bending edge of a hexagon, can then be derived from a trigonometric relationship as

$$
a=2 r \sin ^{-1} \frac{\frac{c}{2}}{r} \approx 0.2032 D
$$

where $D$ is the diameter of the circumscribed sphere of the folded truncated icosahedron.

\section{Finite-element simulations of volatilization induced deformation}

We use a finite-element method to simulate the shape transformation of structures in Figure 3. The total deformation gradient $\boldsymbol{F}$ can be decomposed into elastic deformation gradient $\boldsymbol{F}_{\boldsymbol{e}}$ and pure volume deformation gradient $\boldsymbol{F}_{\boldsymbol{v}}$ induced by volatilization, and can be expressed as

$$
\boldsymbol{F}=\boldsymbol{F}_{e} \boldsymbol{F}_{v}
$$

where $\boldsymbol{F}_{\boldsymbol{v}}$ can be further expressed as $\boldsymbol{F}_{\boldsymbol{v}}=\alpha^{\frac{1}{3}} \boldsymbol{I}$. Here, $\alpha$ is the volume shrinkage ratio induced by volatilization. 
To capture the essence of the reaction conversion dependent material behavior, we use a neo-Hookean hyperelastic model to describe the stress-strain relationship, which can be expressed as:

$$
\boldsymbol{\sigma}=J^{-1}\left(\kappa(\varphi) \ln J \boldsymbol{I}+\mu(\varphi) J^{-\frac{2}{3}}\left(\boldsymbol{B}-\frac{1}{3}(\operatorname{tr} \boldsymbol{B}) \boldsymbol{I}\right)\right)
$$

where $\mathbf{B}=\boldsymbol{F}_{\boldsymbol{e}} \boldsymbol{F}_{\boldsymbol{e}} \boldsymbol{T}, \kappa=\frac{E(\varphi)}{3(1-2 v)}$, and $\mu=\frac{E(\varphi)}{2(1+v)}$ are the left Cauchy-Green deformation tensor, the bulk modulus of the material, shear modulus of the material, respectively. $J$ is the determinant of the deformation gradient $\boldsymbol{F}_{\boldsymbol{e}}$. The finite deformation model above is implemented as a user material subroutine (UMAT) in the commercial software ABAQUS for analysis.

Printing diameters for the shape-morphing structures in the main text

Table S1. List of printing parameters for the shape-morphing structures in the main text

\begin{tabular}{ccccc}
\hline Name & Number & $\begin{array}{c}\text { Sample } \\
\text { thickness } \\
(\mathbf{m m})\end{array}$ & $\begin{array}{c}\text { Irradiation time } \\
\mathbf{( s )}\end{array}$ & $\begin{array}{c}\text { Grayscale } \\
\text { value }\end{array}$ \\
\hline GT logo sheet & Figure 1C & 0.26 & 30 & 0,149 \\
Rectangular strip & Figure 2A-B & 0.19 & $10,15,20,30$ & 149 \\
Rectangular strip & Figure 2C & 0.19 & 20 & $88-227$ \\
2D hexagonal sheet & Figure 2F-G & 0.26 & $5.6,9,11.4$ & 149 \\
Rectangular strip & Figure 3A-D & 0.19 & 16,30 & $80-255$ \\
Spiral strip & Figure 3E & 0.19 & 19 & $80-149$ \\
Twisted ribbon & Figure 3F & 0.26 & 12.5 & 149,255 \\
Saddle-like structure & Figure 3G & 0.26 & 25,25 & 149 \\
Wavy disc & Figure 3H & 0.26 & 10,10 & 149 \\
Flower & Figure 4A & 0.26 & 12 & 149,255 \\
Pop-up structure & Figure 4B & 0.26 & 4,4 & 149,255 \\
Stanford Bunny & Figure 4C & 0.26 & 0.26 & $149,200,255$ \\
Miura-Ori origami & Figure 5B & 0.19 & 4,4 & 149,255 \\
\hline
\end{tabular}

movie S1. Self-folding of Buckyball.

movie S2. Self-folding of a flower. 
movie S3. Self-folding of a Stanford Bunny.

movie S4. Self-folding of a Miura-Ori origami.

1. Kumar, V.; Bhardwaj, Y. K.; Goel, N. K.; Francis, S.; Dubey, K. A.; Chaudhari, C. V.; Sarma, K. S. S.; Sabharwal, S., Coating Characteristics of Electron Beam Cured Bisphenol a Diglycidyl Ether Diacrylate-Co-Aliphatic Urethane Diacrylate Resins. Surface and Coatings Technology 2008, 202 (21), 5202-5209.

2. Sigma-Aldrich, Trimethylolpropane triacrylate, https://www.sigmaaldrich.com/catalog/product/aldrich/246808, accessed: March 2020.

3. Allnex, EBECRYL ${ }^{\circledR} 8402$, https://www.allnex.com/en/product/66f446f9-2010-43d3-8938422e79e3d247/ebecryl-8402, accessed: March 2020.

4. Cabral, J. T.; Hudson, S. D.; Harrison, C.; Douglas, J. F., Frontal Photopolymerization for Microfluidic Applications. Langmuir : the ACS journal of surfaces and colloids 2004, 20, 1002010029.

5. Warren, J. A.; Cabral, J. T.; Douglas, J. F., Solution of a Field Theory Model of Frontal Photopolymerization. Physical review. E, Statistical, nonlinear, and soft matter physics 2005, 72 (2 Pt 1), 021801.

6. Vitale, A.; Hennessy, M. G.; Matar, O. K.; Cabral, J. T., Interfacial Profile and Propagation of Frontal Photopolymerization Waves. Macromolecules 2014, 48 (1), 198-205.

7. Doyle, C. D., Kinetic Analysis of Thermogravimetric Data. Journal of applied polymer science 1961, 5 (15), 285-292.

8. O'Brien, D. J.; Mather, P. T.; White, S. R., Viscoelastic Properties of an Epoxy Resin during Cure. Journal of Composite Materials 2001, 35 (10), 883-904.

9. Zhao, Z.; Wu, J.; Mu, X.; Chen, H.; Qi, H. J.; Fang, D., Desolvation Induced Origami of Photocurable Polymers by Digit Light Processing. Macromolecular rapid communications 2016, 38 (13), 1600625. 